\title{
Sugar palm (Arenga pinnata): Its fibres, polymers and composites.
}

\begin{abstract}
Sugar palm (Arenga pinnata) is a multipurpose palm species from which a variety of foods and beverages, timber commodities, biofibres, biopolymers and biocomposites can be produced. Recently, it is being used as a source of renewable energy in the form of bioethanol via fermentation process of the sugar palm sap. Although numerous products can be produced from sugar palm, three products that are most prominent are palm sugar, fruits and fibres. This paper focuses mainly on the significance of fibres as they are highly durable, resistant to sea water and because they are available naturally in the form of woven fibre they are easy to process. Besides the recent advances in the research of sugar palm fibres and their composites, this paper also addresses the development of new biodegradable polymer derived from sugar palm starch, and presents reviews on fibre surface treatment, product development, and challenges and efforts on properties enhancement of sugar palm fibre composites.
\end{abstract}

Keyword: Polymer-matrix composites (PMCS); Fibres; Mechanical properties; Physical properties 